\title{
Hyperprolactinemia and breast abscess: Is there a link?
}

\author{
Rojbi I, Haouat E, Kandara H, Saada B, Ben Salem L, Ben Slama C \\ National Institute of Nutrition - Tunis- Tunisia
}

Hyperprolactinemia is a common endocrine condition causing galactorrhea. Breast abscess is a frequent complication of the post partum period and breast feeding but it rarely occurs out of these two situations.

We report the case of a patient presenting with tumoral hyperprolactinemia associated with a breast abscess.

\section{Case report}

A 34 years-old patient presented with galacthorrea, spaniomenorrhea and headache for six months. She had nothing special in her medical history and was not on drugs. She was the mother of two children aged 8 and 4 years old. Prolactin assessment was made twice and confirmed the hyperprolactinemia : Prolactin was $94 \mathrm{ng} / \mathrm{ml}$ and $110 \mathrm{ng} / \mathrm{ml}$ indicating of a tumoral etiology. Pituitary MRI concluded to a pituitary microadenoma so that microprolactinoma was diagnosed and the patient put on dopaminergic agonists. Fifteen days later, the patient suddenly developed a painful mass of the left breast. Biologic assessment showed an inflammatory syndrome with hyperleucocytosis. The breast ultrasound examination revealed an abscess of 4 centimeters in the superior external quadrant, with no other abnormalities of the breast. The course of the abscess was good on antibiotics (Amoxicillin + clavulanic acid) and surgery was not indicated.

Discussion

Lactational mastitis is a classic complication of lactation occurring in $2 \%$ to $3 \%$ of lactating women. Breast abscess is seen in $5 \%$ to $11 \%$ of women with mastitis.

Milk stasis is an explicit risk factor for the development of breast inflammation. It occurs typically during lactation but can also occur during hyperprolactinemia with galactorrhea. The association of hyperprolactinemia with breast inflammation and abcess has not been reported in the literature and seems to be rare. In fact, milk stasis is seen in the presence of some predisposing factors such as: insufficient drainage of the breast, rapid weaning, oversupply of milk, pressure on the breast, or feedings that are missed, scheduled, infrequent, or timed during lactation. Other risk factors include blocked ducts and skin breakdown of the nipple (e.g., fissures, cracks, or blisters), although this last factor is neither essential nor sufficient to cause infection.

Milk stasis, rather than the presence of bacteria entering through a break in the skin, may be a major cause of the development of breast infection.

Unlike the majority of the cases described in literature, our case did not refer to a pregnancy or lactation period. The hyperprolactinemia seems to be the only cause since the patient does not present any mechanical risk factor for milk stasis.

Because no other etiology could be found in the young patient for the mammary abscess, the prolactin excess is the most probable cause. Even considering the rarity of the association it is important to point the hormonal role of prolactin in changing the anatomy of the breast.

\section{Conclusion}

This case report highlights the fact that even during pathological hyperprolactinemia, the risk of breast abcess exist. The histology of the breast glands may be modified by a prolactin effect leading to a risk of developing breast abscess. This phenomenon is may be exactly the same than during breastfeeding but further studies are necessary to confirm the hypothesis. No other similar cases have been reported in the literature. 